\title{
Covid-19: Administrative Belange der Intensivmedizin
}

\begin{abstract}
Thierry Fumeaux ${ }^{a}$, Franziska von Arx ${ }^{b}$, Andreas Perren ${ }^{c}$, Mark Kaufmann ${ }^{d}$, Gian-Reto Klegere, Martin Balmer ${ }^{f}$, Hans Parggerg, Jolanda Contartese ${ }^{h}$, Mario Kaufmanni, Constanze Hergeth ${ }^{j}$, Hans Ulrich Rothen ${ }^{k}$

${ }^{a}$ Geschäftsführender Präsident SGI, Nyon; ${ }^{b}$ Präsidentin Pflege SGI, Zürich; ${ }^{c}$ Präsident SGI-Kommission Datensatz, Bellinzona; ${ }^{d}$ Past-Präsident

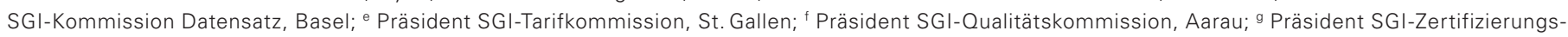

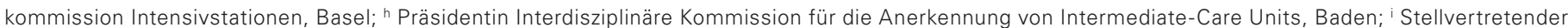
Chef Geschäftsstelle Koordinierter Sanitätsdienst (KSD-SSC), Ittigen; ' Leiterin Geschäftsbereich Akutsomatik, SwissDRG AG, Bern; ${ }^{k}$ SGI-Tarifkommission, SGI-Kommission Datensatz, SGI-Qualitätskommission, Bern
\end{abstract}

Dokumentation von Behandlungskapazität im Informations- und Einsatzsystem des Koordinierten Sanitätsdienstes, Falldokumentation im Minimalen Datensatz der Schweizerischen Gesellschaft für Intensivmedizin und der Intermediate Care sowie Abgeltung der Behandlungsfälle.

\section{Massnahmen zur Sicherstellung der Versorgung von kritisch Kranken im Rahmen der Pandemie}

\subsection{Allgemeine Vorbemerkungen}

- Das vorliegende Dokument behandelt ausschliesslich administrative Belange.

- Grundsätze zur medizinischen Versorgung, inkl. Behandlungskonzepte und Triage-Empfehlungen bei Ressourcenknappheit, werden an anderer Stelle erarbeitet und publiziert (siehe BAG, SAMW, medizinische Fachgesellschaften etc.).

\subsection{Erweiterung der Behandlungskapazität -} Ausgangslage

- Im Normalfall werden in der Schweiz kritisch kranke Patienten, die einer intensivmedizinischen Behandlung bedürfen, in einer von der SGI zertifizierten oder anerkannten Intensivstation behandelt.

- Je nach Schweregrad der akuten Erkrankung ist im Einzelfall auch eine Betreuung in einer anerkannten Intermediate-Care-Station (IMC-Unit) möglich.

- Im Rahmen der aktuellen ausserordentlichen Lage ist es zwingend notwendig, die Behandlungskapazität in Intensivstationen und IMC-Units zu erhöhen. Dazu sind, neben der Bereitstellung von qualifiziertem Personal, Infrastruktur (Behandlungsplätze, Überwachungsgeräte wie Monitore, medizintechnische Apparate zur
Unterstützung von Vitalfunktionen usw.) etc., auch administrative und weitere Belange zu berücksichtigen.

\subsection{Erhöhung der Bettenzahl}

- Die Erhöhung der Bettenzahl ist nach den Vorgaben der zuständigen Organe von Bund und Kanton sicherzustellen.

- Die für den Normalfall gemäss Zertifizierungskommission Intensivstationen (ZK-IS) der SGI respektive der Kommission für die Anerkennung von Intermediate-Care-Units (KAIMC) geltende Bettenzahl wird nachfolgend «zertifizierte Betten" genannt.

- Die zusätzlichen Bettenplätze werden nachfolgend «Behandlungsbetten ad hoc» genannt.

- Die Regelung gilt sowohl für zertifizierte resp. anerkannte Intensivstationen wie auch für anerkannte Intermediate-Care-Units.

\section{Behandlungsbetten ad hoc}

- Patientenbetreuung in Behandlungsbetten ad hoc auf Intensivstationen erfolgt unter Verantwortung der medizinischen Leitung einer zertifizierten oder anerkannten Intensivstation.

- Patientenbetreuung in Behandlungsbetten ad hoc auf IMC-Units erfolgt unter Verantwortung der medizinischen Leitung einer zertifizierten resp. anerkannten Intensivstation oder einer anerkannten Intermediate-Care-Unit. 
- Diese medizinische Verantwortung kann in Ausnahmefällen an entsprechend qualifizierte Fachpersonen delegiert werden. Diese Delegation ist klar zu regeln und schriftlich festzuhalten. Die zuständigen Organe haben Einsichtsrecht in diese Regelungen. Missbrauch kann sanktioniert werden.

- Soweit möglich und sinnvoll sind die Standards gemäss «Richtlinien für die Zertifizierung von Intensivstationen (IS) durch die Schweizerische Gesellschaft für Intensivmedizin (SGI)» vom 3.9.2015 respektive den «Richtlinien zur Anerkennung der Schweizer Intermediate Care (IMC) Units» vom 1.1.2020 einzuhalten. Einschränkungen sind möglich; die Verantwortung tragen die medizinische Leitung der Intensivstation resp. IMC-Unit oder deren vorgesetzte Stellen.

- Soweit möglich und sinnvoll gelten die für den Normalfall festgelegten Behandlungsgrundsätze. Ausnahmen werden an anderer Stelle geregelt (vgl. Punkt 1.1).

\section{Dokumentation: Informations- und Einsatzsystem (IES)}

- Das internetbasierte Informations- und Einsatzsystem (IES) ist ein Instrument des Koordinierten Sanitätsdienstes (KSD) des Bundes.

- Die Dokumentation der Behandlungskapazität im IES ist eine der wesentlichen Grundlagen für die Lageinformation COVID-19 des Sanitätsdienstlichen Koordinationsorgans (SANKO) des KSD.

- Die Leitungen der Intensivstationen und Intermediate-Care-Units sind gehalten, die entsprechenden Informationen jeweils durch den von ihnen bezeichneten Single Point of Contact im IES eintragen zu lassen. Zurzeit werden die Daten im IES einmal pro Tag ausgewertet.

- Für die Dokumentation der Behandlungskapazität (Bettenzahl) und weiterer Informationen im IES gelten die Vorgaben des SANKO. Per 27.3.2020 wurde der Datensatz zwecks differenzierterer Dokumentation der Behandlungskapazität ergänzt. Im Mitgliederbereich der SGI-Website sind unter «COVID-19» die aktuell gültigen Datenfelder zusammengestellt.

- Die Summe der Anzahl zertifizierter (resp. anerkannter) Betten und der Anzahl Behandlungsbetten ad hoc ergibt die Anzahl betriebener Betten. Zusammen mit den anderen Daten im IES ergeben diese Daten wichtige Kennzahlen zu Behandlungskapazität und Auslastung der Spitäler.

\section{Dokumentation: Minimaler Datensatz der SGI (MDSi) und der Intermediate Care (MDSimc)}

- Die Dokumentation der Falldaten im MDSi oder MDSimc muss sichergestellt sein. Die Datenerfassung geschieht im Normalfall kontinuierlich und im Rahmen der Patientenbetreuung. Ist die aufgrund der ausserordentlichen Lage nicht möglich, muss eine nachträgliche Datenerfassung vorgesehen werden.

- Wie bereits vor COVID-19 festgelegt, ist die Erfassung des Sequential-Organ-Failure-Assessment (SOFA)-Scores im MDSi oder MDSimc erwünscht, bleibt aber bis Ende 2020 fakultativ. Über eine allfällige Verlängerung dieser Phase mit fakultativer Erhebung wird zu einem späteren Zeitpunkt entschieden. Für die Dokumentation des SOFA-Scores im Rahmen von SwissDRG gilt die Regelung der SwissDRG AG.

- Ziele der Dokumentation der Falldaten sind:

- Sicherstellen der Nachverfolgbarkeit des Patientenflusses.

- Sicherstellen der Nachverfolgbarkeit der Dokumentation zum summarischen Krankheitsverlauf jedes einzelnen Patienten. Die detaillierte Dokumentation des Krankheitsverlaufes (Krankenakte/Krankengeschichte) wird gemäss den Regelungen der jeweiligen Intensivstationen oder IMC-Units resp. des entsprechenden Spitals sichergestellt.

- Grundlage zur Nachbereitung der Pandemie (administrativ, wissenschaftlich, Qualitätssicherung etc.).

- Um die nachträgliche Analyse der Fälle mit COVID-19 im MDSi/MDSimc zu erleichtern, sollen die nachfolgenden Datenfelder wie folgt ausgefüllt werden:

- Erst-Diagnose (Hauptdiagnose bei Eintritt in die Intensivstation resp. IMC-Unit): «ARDS» (M_Diag1 $=\mathrm{R} 1)$

- Geplanter Eintritt: «nein» (M_PatGrp = «N»)

- Summe aller Schichten mit Isolation muss >0 sein (M_Isolation muss >0 sein). In der Regel wird M_Isolation der Summe aller Schichten des IS/IMC-Aufenthaltes entsprechen.

- Intensivstationen resp. IMC-Units, welche das durch die SGI zur Verfügung gestellte Web-basierte MDSiTool verwenden, können die Dokumentation der Falldaten von auf Behandlungsplätzen ad hoc betreuten Patienten mit diesem Tool dokumentieren.

- Intensivstationen resp. IMC-Units, die ein eigenes Informatiksystem für die Dokumentation der Fall- 
daten benutzen (Patientendatenmanagementsystem [PDMS], Krankenhausinformationssystem [KIS] usw.), müssen für auf Behandlungsplätzen ad hoc betreute Patienten allenfalls eine Erweiterung des Informatiksystems vornehmen. Der Datenexport in das MDSi ist dabei sicherzustellen. Ist dies nicht möglich, muss die Dokumentation durch andere Mittel sichergestellt sein. Die SGI empfiehlt, dafür das durch die SGI zur Verfügung gestellte Web-basierte Tool zu verwenden (vgl. nachfolgenden $\mathrm{Ab}$ schnitt). Andernfalls muss als minimale Dokumentation ein Journal (Papier oder elektronisch, z.B. im Rahmen eines bereits vorhandenen Klinik-Informationssystems) der Behandlungsfälle geführt werden. Ziel des Journals ist es, die Nachbereitung und insbesondere Nacherfassung der Fälle zu einem späteren Zeitpunkt sicherzustellen. Das Journal soll mindestens enthalten:

- Identifikation des Patienten (gemäss Regelung im jeweiligen Spital)

- Datum/Zeit der Aufnahme in die Intensivstation resp. IMC-Unit

- Datum/Zeit der Verlegung aus der Intensivstation

ihre Mitglieder vom

18.3.2020 und

Stellungnahme der SGI

vom 19.3.2020.

2 Initiale Installation re-

mote, d.h. ferngesteuert

zwischen der IT-Abteilung

des Spitals und Protec-

Data.Zeitbedarf:

ca. 2 Stunden.

3 mdsi[at]protecdata.ch oder Tel. 0566778090 (erste Ansprechperson: L. Lanz, sonst M. Amstutz oder C. Kaufmann).

Schweizerische Gesellschaft für Intensivmedizin SGI c/o IMK Institut für Medizin und Kommunikation AG Münsterberg 1

4001 Basel

Telefon: +41615615364

sgi@imk.ch
- Vitalstatus bei Verlegung (lebend/gestorben)

- Anzahl Schichten mit maschineller Beatmung (invasiv und nicht-invasiv; aufgerundet auf ganze Schicht)

- Verlegungsort

- Für das Journal gelten die gleichen Sorgfalts- und Aufbewahrungspflichten wie für die Krankengeschichte.

- Die SGI stellt das Web-basierte Tool allen Intensivstationen resp. IMC-Units zur Verfügung ${ }^{1}$, die im Normalfall mit einem eigenen Informationssystem (Patientendatenmanagementsystem [PDMS], Krankenhausinformationssystem [KIS] usw.) arbeiten, dies aber für Dokumentation der Falldaten von auf Behandlungsplätze ad hoc betreuten $\mathrm{Pa}$ tienten nicht einsetzen können. ${ }^{2}$ Dieses Web-basierte Tool erlaubt Dokumentation im Zwei- oder Dreischichtbetrieb. Ein bereits installiertes Webbasiertes Tool kann ebenfalls von Drei- auf Zwei- schichtbetrieb umgestellt werden. Bitte in diesem Fall den Betreiber der MDSi-Datenbank ProtecData kontaktieren. $^{3}$

\section{Abgeltung der Behandlungsfälle}

- In enger Abstimmung mit der SGI empfiehlt die SwissDRG AG, eine Abrechnung der intensivmedizinischen Komplexbehandlung sowie der Komplexbehandlung in einer Intermediate-Care-Station zeitlich limitiert auch in nicht zertifizierten bzw. nicht anerkannten Räumlichkeiten/Einheiten zu ermöglichen.

- Eine Kodierung gemäss CHOP-Codes 99.B7.1-, 99.B7.2und 99.B7.3- sowie 99.B8.1-, 99.B8.2- und 99.B8.3- und die Abbildung der maschinellen Beatmung ist aufgrund der dynamischen Ausbreitung des SARS-CoV-2 und der damit verbundenen Krankheit COVID-19 auch ausserhalb der Räume einer zertifizierten resp. anerkannten Intensivstation/anerkannten Intermediate-Care-Station unter nachfolgend aufgeführten Bedingungen zulässig:

- Diese Anpassung gilt für eine begrenzte Zeitdauer während der SARS-Cov-2-Pandemie.

- Es handelt sich um eine dokumentierte intensivmedizinische Behandlung resp. Versorgung auf einer Intermediate-Care-Station (gilt nicht für Überwachung bei Verdacht auf SARS-CoV-2-Infektion oder lediglich bei einer Isolation). Es liegt mindestens ein Organversagen vor, welches die Behandlung auf einer Intensivstation/Intermediate-Care-Station notwendig macht.

- Die intensivmedizinischen Leistungen werden inkl. MDSi/MDSimc dokumentiert.

- Die Verantwortung und Leitung der zusätzlichen Kapazitäten erfolgen durch Personal einer zertifizierten resp. anerkannten Intensivstation bzw. einer anerkannten Intermediate-Care-Unit.

- Die Patientenbehandlung findet unter Fachführung und durch Mitbeteiligung von Personal einer zertifizierten resp. anerkannten Intensivstation bzw. einer anerkannten IntermediateCare-Unit statt. 